\title{
PERFIL EPIDEMIOLÓGICO DAS FRATURAS DE FACE: UMA REVISÃO DE LITERATURA
}

\author{
Epidemiological Profile of Face Fractures: a Literature Review
}

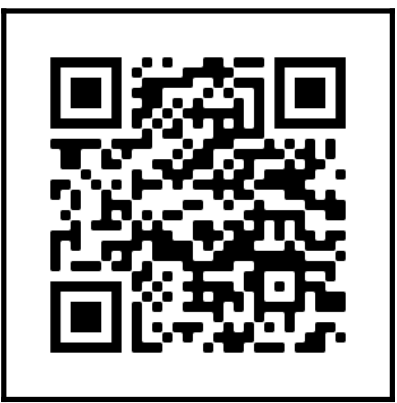

\section{Autores:}

\section{Rodrigo de Souza Viana}

Graduando em odontologia pela Universidade do Grande Rio (UNIGRANRIO), RJ, Brasil.

Acadêmico bolsita pelo Município do Rio de janeiro, lotado no Hospital Municipal Salgado Filho, RJ, Brasil.

\section{Jackeline Nogueira de Paula Barros}

Staff do Serviço de Cirurgia e Traumatologia Bucomaxilofacial do Hospital Municipal

Salgado Filho, Rio de Janeiro, RJ, Brasil

Mestre em Clínica Odontológica pela Universidade Federal do Rio de Janeiro, RJ, Brasil.

Instituição onde o trabalho foi realizado: Hospital Municipal Salgado Filho, RJ, Brasil.

\section{Endereço para correspondência:}

Endereço: Avenida Meriti, 2827 - Vila da Penha, Rio de Janeiro - RJ, 21211-007

Telefone: (21) 96466-5971

E-mail: odontorodrigoviana@gmail.com

\section{RESUMO}

As fraturas de face são um problema de saúde pública que afeta a qualidade de vida dos indivíduos que sofreram esse agravo. Sabendo-se da importância dos estudos epidemiológicos para o enfrentamento desse problema, este trabalho teve como objetivo traçar o perfil epidemiológico das fraturas de face, contribuindo assim, para a elaboração de políticas públicas mais eficazes e tratamento adequado. Para isso foi ralizada uma revisão de literatura nas principais bases de dados, Literatura Latino-Americana e 
do Caribe em Ciências da Saúde(LILACS), Medical Literature Analysis and Retrievel System Online (MEDLINE) e Bibliografia Brasileira de Odontologia (BBO) através da Biblioteca Virtual em Saúde (BVS) e diretamente pela interface da Literature Analysis (PubMed/MEDLINE), nos últimos 20 anos, contemplando populações, culturas e faixas etárias heterogêneas. Observouse que o sexo masculino foi o mais acometido e sua incidência foi maior entre os jovens, as principais etiologias foram às agressões físicas e os acidentes de transito sobretudo com o envolvimento de motocicletas associado ao uso de álcool e drogas. Os ossos nasais e a mandíbula foram as estruturas que sofreram mais fraturas e o tratamento mais utilizado foi a redução fechada. Neste contexto, faz-se necessário medidas ao combate às desigualdades sociais e desemprego, incentivo à educação e intensificação das leis de trânsito.

Palavras-chave: Traumatismo facial; Epidemiologia; Fratura

\section{ABSTRACT}

Face fractures are a public health problem that affects the quality of life of individuals who have suffered this condition. Knowing the importance of epidemiological studies to face this problem, this study aimed to outline the epidemiological profile of facial fractures, thus contributing to the development of more effective public policies and adequate treatment. For this, a literature review was carried out in the main databases, Latin American and Caribbean Literature in Health Sciences (LILACS), Medical Literature Analysis and Retrievel System Online (MEDLINE) and Brazilian Bibliography of Dentistry (BBO) through the Library Virtual Health (VHL) and directly through the Literature Analysis interface (PubMed / MEDLINE), in the last 20 years, covering heterogeneous populations, cultures and age groups. It was observed that the male gender was the most affected and its incidence was higher among young people, the main etiologies were physical aggressions and traffic accidents, especially with the involvement of motorcycles associated with the use of alcohol and drugs. The nasal bones and the mandible were the structures that suffered the most fractures and the most used treatment was closed reduction. In this context, measures are needed to combat social inequality and unemployment, encourage education and intensify traffic laws.

Keywords: Facial trauma; Epidemiology; Fracture 


\section{INTRODUÇÃO}

Os traumas faciais são um grave problema de saúde pública, podendo ter um efeito devastador, principalmente devido as deformidades e cicatrizes causadas na face, que é a parte mais visível e exposta do corpo humano, levando o indivíduo muitas vezes a desenvolver problemas psicológicos como depressão, ansiedade e estresse, conforme o aumento da gravidade das lesões (POKHAREL et al., 2015 e RAMPA et al., 2019). E essa gravidade geralmente é acentuada quando associadas a traumas dentários, ossos, tecidos moles, além de regiões como cérebro, olhos, ouvidos, tórax, abdômen, entre outras (OLUSANYA et al., 2014).

Todas essas condições advindas do trauma de face, principalmente aquelas associadas às fraturas de face podem gerar meses de internação hospitalar, procedimentos cirúrgicos e de reabilitação, demandando assim, um tempo maior de afastamento das atividades laborais, aumento de custo financeiro e grande impacto socioeconômico para toda a sociedade, repercutindo negativamente na qualidade de vida de todos os envolvidos (OLUSANYA et al., e LALOO et al., 2020).

As causas das fraturas de face são variadas, sendo as principais: acidentes de trânsito e agressão (RAMOS et al., 2018). O gênero mais acometido é o masculino, quando comparado ao feminino (SAMIEIRAD et al., 2015 e RAMOS et al., 2018. E as fraturas dos ossos nasais e mandíbula foram as mais incidentes (LUCENA et al., 2016)

Neste contexto, estudos epidemiológicos com enfoque nas fraturas faciais é de suma relevância, a medida que auxiliam nas estratégias de educação e prevenção, abordagem terapêutica adequada e políticas públicas eficientes(LUCENA et al.,2016).

\section{MATERIAL E MÉTODOS}

Trata-se de uma revisão de literatura sobre a epidemiologia das fraturas faciais, visando realizar um levantamento bibliográfico atual nas principais bases de dados: LILACS, MEDLINE e BBO-odontologia através da Biblioteca Virtual em Saúde (BVS) e diretamente pela interface da Literature Analysis (PubMed/MEDLINE).

Foram utilizadas as palavras-chave:Traumatismo facial, Epidemiologia e Fratura na língua portuguesa e Facial trauma, Epidemiology and Fracture 
na língua inglesa, sendo utilizado o operador booleano "AND" em combinação entre as palavras-chaves.

Como critério de inclusão cita-se: artigos publicados entre os anos 2001 a 2021 (últimos 20 anos), em português e inglês, com textos completos e gratuitos e enfoque no perfil epidemiológico dos traumas de face. Já os critérios de exclusão foram: as revisões de literatura, relato de caso, artigos que ultrapassem o tempo determina do nos critérios de inclusão e os que não tiveram relação com a área de Buco maxilo facial.

Após busca, encontrou-se o total de 518 artigos, os quais foram inicialmente selecionados pelos títulos, refinados pelos resumos e depois lidos integralmente, restando no final apenas 20 artigos, segundo os critérios de inclusão/exclusão, variantes necessárias e remoção de duplicatas, conforme figura 1.

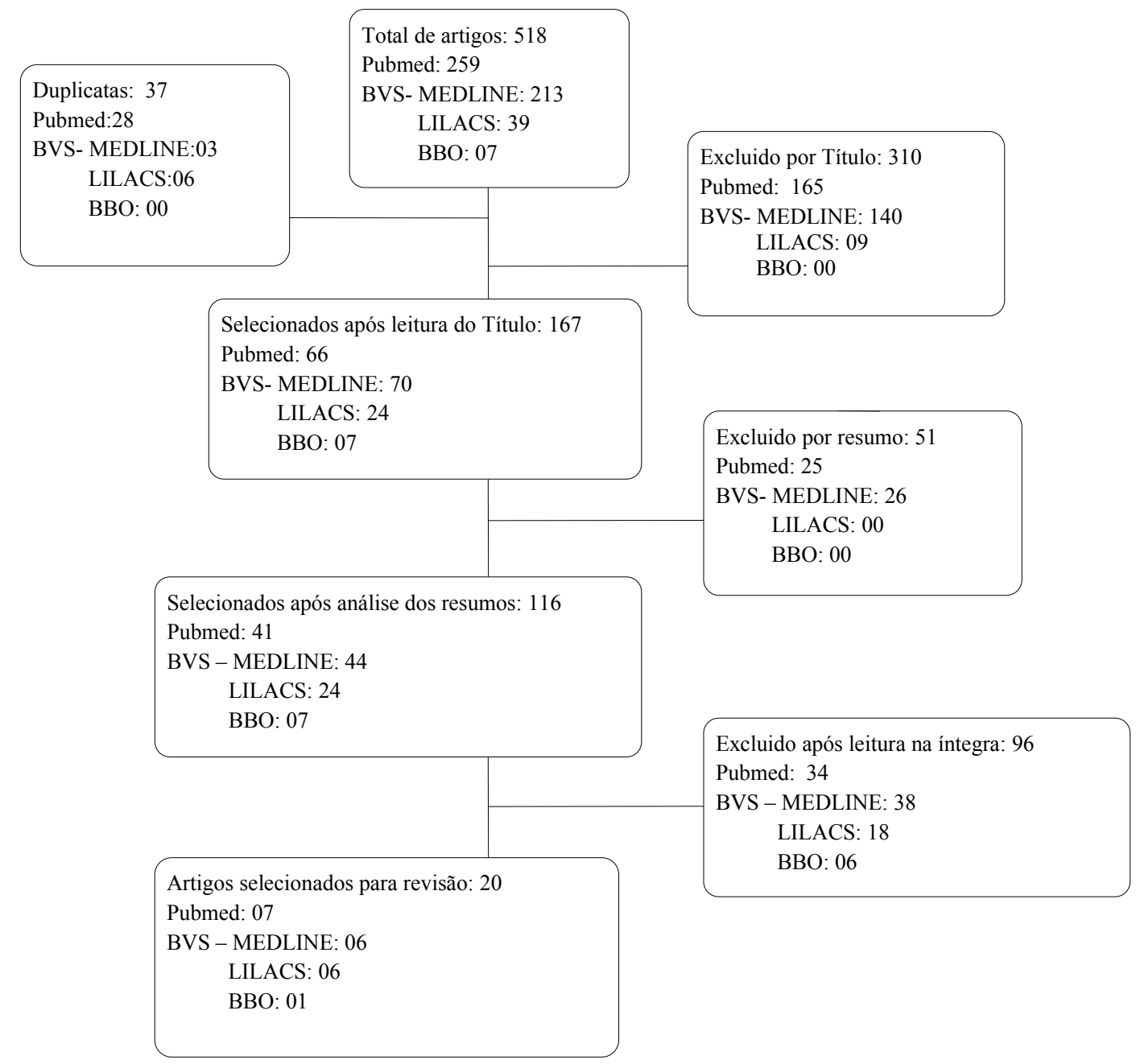

Figura 1: Fluxograma dos artigos selecionados. 


\section{RESULTADOS}

Foram encontrados 474 artigos retirando as duplicatas, e após considerar os critérios de inclusão e exclusão e as variantes necessárias restaram para análise apenas 20 artigos. Na tabela 1 estão apresentados os artigos e respectivos autores, o período de publicação dos artigos e local onde foram desenvolvidas as pesquisas.

Ainda foram utilizadas como variantes deste estudo: Etiologia mais prevalente em fraturas de face, Fatores predisponentes e facilitadores, que influenciam para que tais fraturas ocorram com maior frequência (idade, gênero, ingestão de álcool e drogas ilícitas, entre outros), as fraturas mais comumente observadas e o tratamento mais utilizado.

A maior parte foi publicada em inglês (12 artigos), ainda que tenham sido estabelecidos como critério para a busca, artigos publicados em português e inglês.

Tabela 1: Autor, ano e local de produção do artigo, etiologia das fraturas de face mais prevalente, fatores predisponentes, tipos de fratura de face e tratamento.

\begin{tabular}{|c|c|c|c|c|c|}
\hline $\begin{array}{c}\mathbf{N} \\
0\end{array}$ & $\begin{array}{c}\text { Autor/ Ano/ } \\
\text { Local }\end{array}$ & Etiologia & $\begin{array}{c}\text { Fatores } \\
\text { Predisponentes }\end{array}$ & $\begin{array}{c}\text { Fraturas } \\
\text { Observadas }\end{array}$ & Tratamento \\
\hline 01 & $\begin{array}{c}\text { MUKHOPADYAY } \\
\text { et al } \\
2020 \\
\text { Índia } \\
\text { (Kolkata) }\end{array}$ & $\begin{array}{l}\text { Quedas/acidentes } \\
\text { de trânsito }\end{array}$ & $\begin{array}{l}\text { Meninos/ } \\
\text { ligeiramente mais } \\
\text { alto de } 7 \text { a } 12 \text { anos. }\end{array}$ & $\begin{array}{l}\text { mandíbula/ossos } \\
\text { nasais }\end{array}$ & não relataram \\
\hline 02 & $\begin{array}{c}\text { VLAVONOU et al } \\
2018 \\
\text { França }\end{array}$ & Quedas & $\begin{array}{l}\text { Mulheres de } 75 \text { a } \\
85 \text { anos/ } \\
\text { deficiência } \\
\text { cognitiva }\end{array}$ & Ossos nasais & Não relataram \\
\hline 03 & $\begin{array}{c}\text { SAMIEIRAD et al } \\
2015 \\
\text { Irã } \\
\text { (sudeste) }\end{array}$ & $\begin{array}{l}\text { Acidentes de } \\
\text { trânsito/ lesões } \\
\text { ocupacionais }\end{array}$ & $\begin{array}{l}\text { Homens de } 20 \text { a } 30 \\
\text { anos/ baixas } \\
\text { condições sócio } \\
\text { econômicas/ } \\
\text { consumo de álcool } \\
\text { e drogas/ } \\
\text { aumentam no } \\
\text { verão e no outono }\end{array}$ & $\begin{array}{l}\text { Mandíbula/ ossos } \\
\text { nasais }\end{array}$ & redução fechada \\
\hline 04 & $\begin{array}{l}\text { RAMOS et al } \\
2018 \\
\text { Brasil } \\
\text { (Paraíba) }\end{array}$ & $\begin{array}{l}\text { Acidentes de } \\
\text { trânsito } \\
\text { (motocicleta)/ } \\
\text { agressão física }\end{array}$ & $\begin{array}{l}\text { Homens de } 20 \text { a } 29 \\
\text { anos/ consumo de } \\
\text { álcool/ aceso às } \\
\text { armas de fogo/ } \\
\text { baixas condições } \\
\text { socioeconômicas }\end{array}$ & $\begin{array}{l}\text { Ossos nasais/ osso } \\
\text { zigomático }\end{array}$ & Não relataram \\
\hline
\end{tabular}




\begin{tabular}{|c|c|c|c|c|c|}
\hline 05 & $\begin{array}{c}\text { KRAFT et al } \\
2012 \\
\text { Áustria } \\
\text { (Innsbruck) }\end{array}$ & $\begin{array}{l}\text { Atividades diárias/ } \\
\text { atividades } \\
\text { esportivas }\end{array}$ & $\begin{array}{l}\text { Homens de } 20 \text { a } 40 \\
\text { anos }\end{array}$ & Osso nasal/ orbitais & Não relataram \\
\hline 06 & $\begin{array}{c}\text { PARK et al } \\
2020 \\
\text { Coréia }\end{array}$ & Não relataram & $\begin{array}{l}\text { Homens de } 0 \text { a } 19 \\
\text { anos é maior } \\
\text { incidência. }\end{array}$ & $\begin{array}{l}\text { Ossos } \\
\text { nasais/orbitais }\end{array}$ & Não relataram \\
\hline 07 & $\begin{array}{l}\text { SBORDONE et al } \\
2018 \\
\text { Itália } \\
\text { (Sul) }\end{array}$ & $\begin{array}{l}\text { Agressão física/ } \\
\text { acidentes de } \\
\text { trânsito }\end{array}$ & $\begin{array}{l}\text { Homens } 30 \text { a } 40 \\
\text { anos }\end{array}$ & $\begin{array}{l}\text { Mandíbula/ } \\
\text { processo } \\
\text { zigomático }\end{array}$ & Não relataram \\
\hline 08 & $\begin{array}{c}\text { RAMPA et al } \\
2019 \\
\text { Estados Unidos Da } \\
\text { América } \\
\text { (Califórnia) }\end{array}$ & $\begin{array}{l}\text { Agressão física } \\
\text { (assaltos)/ quedas }\end{array}$ & $\begin{array}{l}\text { Homens de } 24 \text { a } \\
44 \text { anos }\end{array}$ & $\begin{array}{l}\text { Ossos nasais/ } \\
\text { orbitais }\end{array}$ & Não relataram \\
\hline 09 & $\begin{array}{c}\text { LALLOO et al } \\
2020 \\
\text { Global }\end{array}$ & $\begin{array}{l}\text { Queda/ Agressão } \\
\text { física (violência) }\end{array}$ & $\begin{array}{l}\text { Homens de } 05 \text { a } 20 \\
\text { anos e volta a } \\
\text { aumentar após os } \\
70 \text { anos/ regiões } \\
\text { que sofrem } \\
\text { confliteo e } \\
\text { terrorismo/ } \\
\text { consumo de álcool }\end{array}$ & Não relataram & Não relataram \\
\hline 10 & $\begin{array}{c}\text { YKEDA et al } \\
2012 \\
\text { Brasil } \\
\text { (Curitiba) }\end{array}$ & $\begin{array}{l}\text { Agressão } \\
\text { física(violência } \\
\text { sem arma de fogo e } \\
\text { violência com } \\
\text { arma de fogo)/ } \\
\text { quedas }\end{array}$ & $\begin{array}{l}\text { Homens } 20 \text { a } 39 \\
\text { anos/ consumo d } \\
\text { álcool e drogas }\end{array}$ & $\begin{array}{l}\text { Ossos nasais/ } \\
\text { mandíbula }\end{array}$ & Não relataram \\
\hline 11 & $\begin{array}{c}\text { OLUSANYA et } \\
\text { al } \\
2014 \\
\text { Nigéria }\end{array}$ & $\begin{array}{l}\text { Acidentes de } \\
\text { trânsito } \\
\text { (motocicleta)/ } \\
\text { agressão física } \\
\text { (assalto a mão } \\
\text { armada) }\end{array}$ & $\begin{array}{l}\text { Homens com } \\
\text { média de } 32,2 \\
\text { anos }\end{array}$ & $\begin{array}{l}\text { Mandíbula/ } \\
\text { complexo } \\
\text { zigomático }\end{array}$ & $\begin{array}{l}\text { Redução fechada e } \\
\text { imobilização }\end{array}$ \\
\hline 12 & $\begin{array}{c}\text { SANTOS, } \\
\text { ALMEIDA E } \\
\text { SILVA } \\
2013 \\
\text { Brasil } \\
\text { (Salvador,Bahia) }\end{array}$ & $\begin{array}{l}\text { Agressão física/ } \\
\text { acidentes de } \\
\text { trânsito }\end{array}$ & $\begin{array}{l}\text { Mulheres com } \\
\text { média de } 33 \text { anos/ } \\
\text { pardo/ baixa } \\
\text { escolaridade }\end{array}$ & Não relatam. & Não relataram \\
\hline 13 & $\begin{array}{c}\text { WULKAN et al } \\
2005 \\
\text { Brasil } \\
\text { (São Paulo) }\end{array}$ & $\begin{array}{l}\text { Agressão física/ } \\
\text { queda }\end{array}$ & $\begin{array}{l}\text { Homens de } 20 \text { a } 39 \\
\text { anos }\end{array}$ & $\begin{array}{l}\text { Mandíbula/ Le Fort, } \\
\text { pan facial e } \\
\text { complexas }\end{array}$ & Não relataram \\
\hline 14 & $\begin{array}{l}\text { FAVERANI et al } \\
2009 \\
\text { Brasil } \\
\text { (Araçatuba, São }\end{array}$ & $\begin{array}{l}\text { Agressão física/ } \\
\text { acidentes } \\
\text { ciclísticos }\end{array}$ & $\begin{array}{l}\text { Homens de } 21 \text { a } 25 \\
\text { anos/ consumo de } \\
\text { álcool }\end{array}$ & $\begin{array}{l}\text { Ossos nasais/ } \\
\text { zigomático }\end{array}$ & Não relataram \\
\hline
\end{tabular}




\begin{tabular}{|c|c|c|c|c|c|}
\hline & Paulo) & & & & \\
\hline 15 & $\begin{array}{c}\text { JÚNIOR, KEIM E } \\
\text { HELENA } \\
2010 \\
\text { Brasil } \\
\text { (Blumenau, Santa } \\
\text { Catarina) }\end{array}$ & $\begin{array}{l}\text { Agressão física/ } \\
\text { acidentes de } \\
\text { trânsito }\end{array}$ & $\begin{array}{l}\text { Homens solteiros } \\
\text { de } 20 \text { a } 29 \text { anos/ } \\
\text { com emprego fixo/ } \\
\text { baixa escolaridade }\end{array}$ & $\begin{array}{l}\text { Mandíbula/ ossos } \\
\text { nasais }\end{array}$ & Não relataram \\
\hline 16 & $\begin{array}{l}\text { ALVES et al } \\
2014 \\
\text { Portugal } \\
\text { (Porto) }\end{array}$ & $\begin{array}{l}\text { Acidentes de } \\
\text { trânsito/ acidentes } \\
\text { de trabalho }\end{array}$ & $\begin{array}{l}\text { Homens de } 30 \text { a } 39 \\
\text { anos }\end{array}$ & $\begin{array}{l}\text { Complexo NOE/ } \\
\text { maxilares }\end{array}$ & Não relataram \\
\hline 17 & $\begin{array}{c}\text { FARNEZE et al } \\
2016 \\
\text { Brasil } \\
\text { (Rio de Janeiro) }\end{array}$ & $\begin{array}{l}\text { Acidentes de } \\
\text { trânsito/ agressão } \\
\text { física }\end{array}$ & $\begin{array}{l}\text { Homens com } 33,7 \\
\text { anos em média }\end{array}$ & Mandíbula/ zigoma & Não relataram \\
\hline 18 & $\begin{array}{l}\text { LUCENA et al } \\
2016 \\
\text { Brasil } \\
\text { (Paraíba) }\end{array}$ & $\begin{array}{l}\text { Acidentes de } \\
\text { trânsito/ agressão } \\
\text { física }\end{array}$ & $\begin{array}{l}\text { Homens de } 21 \text { a } 30 \\
\text { anos/consumo de } \\
\text { álcool e drogas/ } \\
\text { aumentam nos } \\
\text { finais de semana e } \\
\text { feridos }\end{array}$ & $\begin{array}{l}\text { Ossos nasais/ } \\
\text { zigoma }\end{array}$ & $\begin{array}{l}\text { Redução aberta e fixação } \\
\text { com miniplacas e } \\
\text { parafusos }\end{array}$ \\
\hline 19 & $\begin{array}{c}\text { FALCÃO, } \\
\text { SEGUNDO E } \\
\text { SILVEIRA } \\
2005 \\
\text { Brasil } \\
\text { (Recife, } \\
\text { Pernambuco) }\end{array}$ & $\begin{array}{l}\text { Acidentes de } \\
\text { trânsito/ agressão } \\
\text { física }\end{array}$ & $\begin{array}{l}\text { Homens e } 21 \text { a } 30 \\
\text { anos/ aumentam } \\
\text { nos finais de } \\
\text { semana, ferido e } \\
\text { na época de festas } \\
\text { típicas }\end{array}$ & $\begin{array}{l}\text { Mandíbula/ } \\
\text { zigomático }\end{array}$ & Não relataram \\
\hline 20 & $\begin{array}{c}\text { NETO at al } \\
2014 \\
\text { Brasil } \\
\text { (Distrito Federal) }\end{array}$ & $\begin{array}{l}\text { Agressão física/ } \\
\text { quedas }\end{array}$ & $\begin{array}{l}\text { Homens de } 21 \text { a } 30 \\
\text { anos/ desemprego/ } \\
\text { tendência a queda } \\
\text { econômica }\end{array}$ & $\begin{array}{l}\text { Ossos nasais/ } \\
\text { zigomático }\end{array}$ & Redução fechada \\
\hline
\end{tabular}

\section{DISCUSSÃO}

Foram selecionados 20 artigos de diferentes localidades do mundo, apresentando populações, culturas e idades heterogêneas. Foram 04 artigos da Europa, 01 artigo da África, 03 artigos da Ásia, 11 artigos das Américas e 01 artigo Global.

A maioria dos autores demonstram que o gênero masculino é o mais prevalente para as fraturas faciais, quando comparados ao feminino, sendo justificado pela natureza mais violenta, principalmente quando envolvidos em atividades que oferecem risco de vida, esportes e violência (SAMIEIRAD et al., 2015). 
Estudos de YKEDA et al., 2012 chamam atenção para a associação entre as questões sociais, culturai e de gênero ao afirmar que essa prevalência pode aumentar quando existe consumo de álcol e drogas ilícitas, o que é corroborado por FAVERANI et al., 2009.

Já SANTOS e ALMEIDA et al., 2013 apontam as mulheres como as mais atingidas por esse agravo, demonstrando que esse panorama é uma tendência mundial, pois acreditam que elas estão em maior número na direção dos automóveis e sofrem violência doméstica. RAMOS et al., 2018 e PARK et al., 2010, reforçam que atualmente a mulher está cada vez mais presente nas atividades sociais, o que era atribuído mais aos homens, aumentando o risco de acidentes.

No que diz respeito à faixa etária, os trabalhos apontam uma prevalência entre os jovens. SANTOS, ALMEIDA E SILVA 2013 e PARK et al., 2020, justificam que os jovens são mais ativos e mais descuidados, pouco maduros, tem comportamento inconsequente e se colocam em situações de risco, SAMIEIRAD et al., 2015 e SBORDONEet al., 2018, concordam e explicam que os jovens transgridem as regras, apresentam uma direção insegura ultrapassando os milites de velocidade, tem tendência a prática exercícios perigosos e se envolvem em conflitos que resultam em violência interpessoal.

Em relação às etiologias foram observadas a agressão física e os acidentes de trânsito como as causas de fraturas faciais mais citadas na literatura consultada. Os autores argumentam que a agressão física está associada a violência urbana em regiões com grande desigualdade social e econômica (SAMIEIRAD et al.,2015), somado ao uso de álcool, drogas e acesso às armas de fogo (RAMOS et al., 2018), desemprego, tendência a queda econômica e crescimento demográfico descontrolado (OLUSANYA et al., 2014 e NETO et al., 2014), baixa escolaridade com primeiro grau completo ou incompleto (SANTOS, ALMEIDA E SILVA 2013 e JÚNIOR, KEIM E HELENA 2010). SBORDONE et al., 2018, acrescentam que a imigração ilegal pode ter aumentado os acidentes por violência interpessoal e LALLOO et al., 2020 trazem o fato que o norte da África e a região do oriente médio são marcados pelo conflito e ataques terroristas que também são responsáveis por fraturas em ambos os sexos.

A segunda etiologia mais prevalente nos estudos foram os acidentes de trânsito que já ocuparam primeiro lugar, Ykeda et al. (2010) justificam a redução dessa prevalência devido a políticas públicas que visam aumentar 
- controle do excesso de velocidade, o uso do cinto de segurança, a proibição de dirigir alcoolizado e a introdução de air bags e barras laterais especialmente importantes na redução de fraturas de face. Porém em contraponto OLUSANYA et al., 2014 ponderam que os acidentes de trânsito ainda são muito expressivos em países mais pobres que possuem as motocicleta como principal meio de transporte pois tem baixo custo e podem ser utilizados para o trabalho porque movem-se rápido dentro das cidades, muitos pilotos são jovens desrespeitam a leis de trânsito e principalmente não usam capacete.

As quedas possuem um papel importante nas causas de fraturas de face e atingem mais os extremos de idade, tem uma predominância de 60 a 79 anos de acordo com LALLOO et al., 2020, acima dos 85 anos no estudo de VLAVONOU et al., 2018 e acima de 70 anos como averiguou LALOO et al., 2020. Os idosos tem a locomoção e o equilíbrio comprometidos devido ao mecanismo fisiológico de propriocepção alterado e associado a algumas infecções oportunistas (YKEDA et al.,2010). No trabalho de LALLOO et al., 2020, acrescentam que esta população faz uso de medicamentos, tem maior tendência a deficiência visual e abuso do álcool.

As crianças apresentam mais queda de acordo com NETO et al., 2020, devido a falta de coordenação motora e equilíbrio em desenvolvimento e o desconhecimento de limites dos seus atos.

O estudo de PARK et al., 2020 apresentou uma limitação quanto a etiologia pois a Coréia possui leis de proteção das informações pessoais das vítimas dos acidentes automobilísticos, por isso não abordou o assunto.

No que tange os ossos mais acometidos pelas fraturas foi evidenciado um predomínio dos ossos nasais e mandíbula. Em conformidade com FAVERANlet al., 2009 E LUCENA et al.,2016, os ossos nasais são mais afetados devido a proeminência do nariz, relativamente desprotegido e em sua anatomia tem pouca cobertura de tecido mole.

FALCÃO, SEGUNDO E SILVEIRA 2005, justificam que a mandíbula é o único osso móvel da face sendo assim estaria mais vulnerável aos fortes impactos.

Demonstrando a sazonalidade da ocorrência das fraturas de face em países com estações do ano mais definidas citam que no verão e no outono as pessoas estão mais expostas as atividades ao ar livre (SAMIEIRAD et al., 2015). No Brasil, os casos são mais observados durante os finais de 
semana, períodos de festas típicas e férias, momentos nos quais as pessoas estão com tempo livre para lazer e consomem mais bebida alcoólica em consonância com LUCENA et al., 2016 e FALCÃO, SEGUNDO E SILVEIRA 2005.

No que diz respeito ao diagnóstico das fraturas, PARK et al., 2020 dizem que a popularização da tomografia computadorizada viabilizou uma análise mais detalhada em relação aos subtipos das fraturas foi confirmado por OLUSANYA et al., 2014 enfatizam que por falta de recursos utilizaram mais a radiografia simples para diagnóstico e isso pode ter levado a não detecção precoce das fraturas de ossos nasais.

De acordo com as referências pesquisadas no tocante aos tipos de tratamento das fraturas foi constatado a abordagem escassa ao assunto. No estudo de SAMIEIRAD et al., 2015, o tratamento prevalente foi a redução fechada com uso da barra de Erich e fixações interdentais, porém muitos cirurgiões preferem redução aberta com placa e parafuso devido a maior estabilidade, precisão anatômica e recuperação imediata da função, no entanto não se popularizou devido aos altos custos, OLUSANYA et al., 2014 corroboram dizendo que devido as restrições logísticas e baixos recursos a redução fechada e imobilização foi o tipo mais comum de tratamento.

No entanto LUCENA et al., 2016, diz em que o manejo terapêutico é orientado pela estrutura óssea afetada, gravidade da lesão e a tecnologia disponível e nesse estudo o tratamento de escolha foi a redução aberta e fixação com miniplaca, já os casos de fratura nasal tiveram uma tendência para o tratamento conservador, porém NETO et al., 2014, relatam que a redução incruenta com uso de pinça de Asch para as fraturas de ossos nasais foi o tratamento de escolha.

Dentre as lesões associadas às fraturas de face, as lesões cerebrais foram as mais vistas nos periódicos consultados, LUCENA et al., 2016, mostram que dos 718 pacientes com fraturas faciais, 136 desenvolveram algum tipo de complicação e desses $67,7 \%$ apresentaram problemas cranianos e cerebrais, segue informando que essa intercorrência pode existir devido a proximidade do cérebro com o rosto $e$ as cargas de choque dissipadas durante o trauma para o neurocrânio. No trabalho de ALVES et al., 2014, pode ser inferido que dos pacientes atendidos $86,6 \%$ tinham ferimentos na cabeça e foram diagnosticados com lesão cerebral. KRAFT et al., 2012, ratificam mostrando que em seu estudo de um total de 2250 pacientes, 2187 sofreram lesões neurológicas. MUKHODADYAY et al., 2020, comentam que 
em seu estudo na população pediátrica das 18 lesões associadas, 05 eram de traumatismo craniano.

\section{CONCLUSÃO}

Diante do exposto, observa-se que a agressão física e o acidente de trânsito foram as principais causas das fraturas de face, os homens jovens foram a população mais prevalente, os ossos nasais e a mandíbula foram as estruturas anatômicas mais acometidas pelas fraturas, sendo a redução fechada e imobilização o método de tratamento mais utilizado.

É importante ressaltar os fatores condicionantes e determinantes de saúde como predisponentes a fratura de face, bem como a urgência no combate às desigualdades sociais e desemprego, incentivo à educação nas escolas e comunidades.

Além disso, amplificar as leis de trânsito, desencorajar o uso de bebida alcoólica e drogas para quem vai dirigir e intensificar o uso dos equipamentos de proteção como capacete e cinto de segurança se fazem necessário, bem como programas de prevenção de quedas para população idosa e pediátrica.

\section{REFERÊNCIAS BIBLIOGRÁFICAS}

1. Mukhopadhyay, Santanu, et al. "Oral and maxillofacial injuries in chidren: a retrospective study." J. Korean Assoc Oral Maxillof Surg 2020; 46: 183190.

2. Vlavonou, Sandrine, et al. "Epidemiology of facial fractures in the elderly." JPRAS Open 16(2018) 84-92.

3. Samieirad, Sahand, et al. "Retrospective study maxillofacial fractures epidemiology and treatment plans in southeast of Iran." Med Oral Patol Oral Cir Bucal. 2015 nov. 1; 20(6): e729-36.

4. Ramos, Joab Cabral, et al. "Estudo epidemiológico do trauma bucomaxilofacial em um hospital de referência da Paraíba." Rev Col Bras Cir 45(6):e1978. 
5. Kraft, Anna, et al. "Craniomaxillofacial Trauma: Synopsis of 14,654 Cases with 35,129 Injuries in 15 Years." Craniomaxillofac Trauma Reconstruction 2012;5:41-50.

6. Park, Cheol-Heum, et al. "Big Data Statistical Analysis of Facial Fractures in Korea." J Korean Med Sci. 2020 Feb 24;35(7):e57.

7. Sbordone, Carolina, et al."The Influence of Socioeconomic Factors on the Epidemiology of Maxillofacial Fractures in Southern Italy." J Craniofac Surg 2018;00: 00-00.

8. Rampa, Sankeerth, et al. "Patient Characteristics and Causes of Facial Fractures in the State of California." J Oral Maxillofac Surg:1-12, 2019.

9. Lalloo, Ratilal, et al."Epidemiology of facial fractures: incidence, prevalence and years lived with disability estimates from the Global Burden of Disease 2017 study." Inj Prev 2020;26:i27-i35.

10. Ykeda, Renier Barreto Arrais, et al. "Perfil epidemiológico de 277 pacientes com fraturas faciais atendidos no pronto atendimento, pelo Serviço de Otorrinolaringologia do Hospital do Trabalhador em Curitiba/PR, no ano de 2010." Int. Arch. Otorhinolaryngol. 2012;16(4):437-444.

11. Olusanya, Adeola Adenike, et al. "Updates on the Epidemiology and Pattern of Traumatic Maxillofacial Injuries in a Nigerian University Teaching Hospital: A 12-Month Prospective Cohort In-Hospital Outcome Study." Craniomaxillofac Trauma Reconstruction 2015;8:5058.

12. Santos, M. S., Almeida, T. F. e Silva, R. A. "Traumas faciais: perfil epidemiológico com ênfase nas características sociais e demográficas e características das lesões, Salvador, BA, 2008." Revista Baiana de Saúde Pública. v.37, n.4, p.1003-1014 out./dez. 2013.

13. Wulkan, Marcelo, et al. "Epidemiologia do trauma facial." Rev Assoc Med Bras 2005; 51(5): 290-5. 
14. Faverani, Leonardo Perez, et al. "Traumas faciais: estudo retrospectivo de 1190 casos na região de Araçatuba." Rev. Bras. Cir. Cabeça Pescoço, v. 38 , n 1, p.22-25, janeiro / fevereiro / março 2009.

15. Júnior, J. C. M., Keim, F. S. e Helena, E. T. S. "Aspectos Epidemiológicos dos Pacientes com Traumas Maxilofaciais Operados no Hospital Geral de Blumenau, SC de 2004 a 2009." Arq. Int. Otorrinolaringol., São Paulo - Brasil, v.14, n.2, p. 192-198, Abr/Mai/Junho -2010.

16. Alves, La-Salete, et al. "Pattern of Maxillofacial Fractures in Severe Multiple Trauma Patients: A 7-year Prospective Study." Brazilian Dental Journal (2014) 25(6): xx-xx.

17. Farneze, Renan de Barros, et al. "Study of the causes of facial fractures in a reference center in Rio de Janeiro, Brazil from 20032012." Dental Traumatology 2016.

18. Lucena, Amanda Lira Rufino de, et al. "Epidemiological Profile of Facial Fractures and Their Relationship With Clinical-Epidemiological Variables." The Journal of Craniofacial Surgery,Volume 27, Number 2, March 20.

19. Facão, M. F. L., Segundo, A. V. L. e Silveira, M. M. F. "Estudo epidemiológico de 1758 fraturas faciais tratadas no Hospital da Restauração, Recife/PE." Rev. Cir. Traumatol. Buco-Maxilo-Fac., Camaragibe v.5, n.3, p. 65-72, julho/setembro 2005.

20. Neto, Altino Vieira de Rezende Filho, et al. "Epidemiologia da fratura de face de pacientes atendidos no pronto socorro de cirurgia plástica do Distrito Federal." Rev. Bras. Cir. Plást. 2014;29(2):227-31.

21. Pokhrerl, Prenit Kumar, et al. "Assessing psychological consequences following maxillofacial trauma using DASS scale-our experience." Asian Journal of Medical Sciences, Mar-Apr 2016, Vol 7 , Issue 2. 